\title{
Erratum to: Electromagnetic shielding effectiveness and mechanical properties of graphite-based polymeric films
}

\author{
G. Kenanakis ${ }^{1}$ - K. C. Vasilopoulos ${ }^{1} \cdot$ Z. Viskadourakis ${ }^{2} \cdot$ N.-M. Barkoula ${ }^{3}$. \\ S. H. Anastasiadis ${ }^{1,4}$ - M. Kafesaki ${ }^{1,5}$ - E. N. Economou ${ }^{1}$ - C. M. Soukoulis ${ }^{1,6}$
}

Published online: 30 August 2016

(C) Springer-Verlag Berlin Heidelberg 2016

\section{Erratum to: Appl Phys A (2016) 122:802 DOI 10.1007/s00339-016-0338-7}

The original version of this article unfortunately contained a mistake. The presentation of Figs. 1 and 2 was incorrect. The correct Figs. 1 and 2 are given here.

The original article was corrected.

The online version of the original article can be found under doi:10.1007/s00339-016-0338-7.

G. Kenanakis

gkenanak@iesl.forth.gr

1 Institute of Electronic Structure and Laser, Foundation for Research \& Technology-Hellas, N. Plastira 100, 70013 Heraklion, Crete, Greece

2 Crete Center for Quantum Complexity and Nanotechnology, University of Crete, 71003 Heraklion, Greece

3 Department of Materials Engineering, University of Ioannina, 45110 Ioannina, Greece

4 Department of Chemistry, University of Crete, 71003 Heraklion, Crete, Greece

5 Department of Materials Science and Technology, University of Crete, 71003 Heraklion, Crete, Greece

6 Ames Laboratory, U.S. Department of Energy and Department of Physics and Astronomy, Iowa State University, Ames, IA 50011, USA

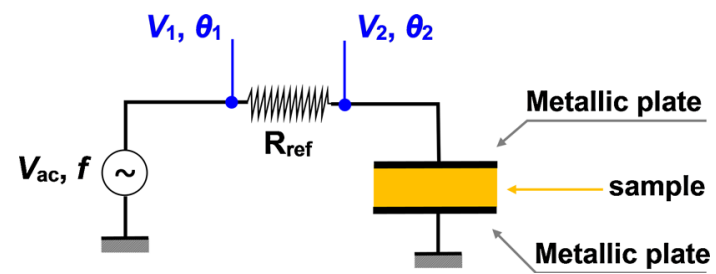

Fig. 1 Experimental setup used for the impedance measurements of the graphite/PS composites

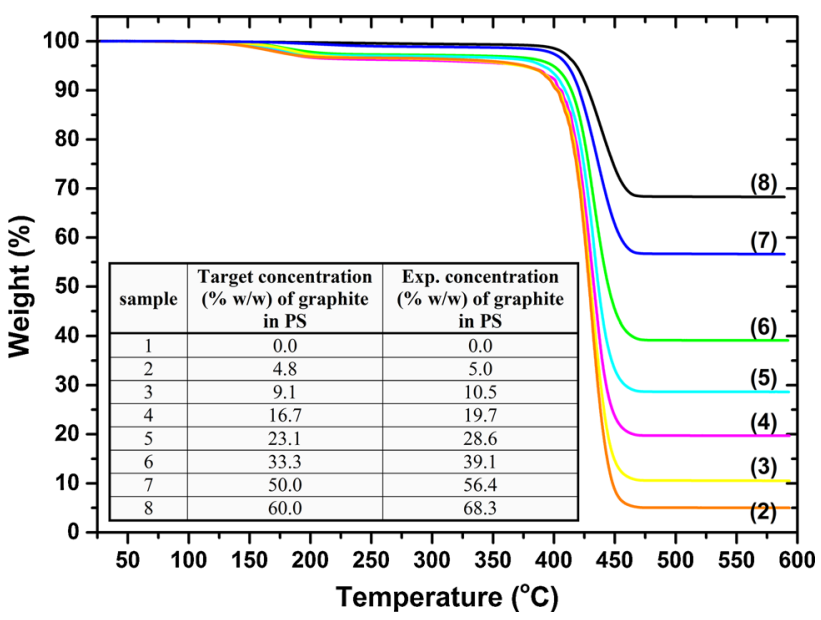

Fig. 2 TGA curves of the graphite/PS composites from RT to $600{ }^{\circ} \mathrm{C}$ at a rate of $20{ }^{\circ} \mathrm{C} / \mathrm{min}$, under air flow. In the inset of Fig. 1, one can see the theoretical and experimental \% w/w concentration of graphite in the polymeric films 